\title{
Persian Character Recognition Using New HYBRIDIZATION OF INDEPENDENT ORTHOGONAL MOMENTS
}

\author{
FatanehAlavipour $^{1}$ and Ali Broumandnia ${ }^{2}$ \\ ${ }^{1}$ Department of Electrical, Computer and Biomedical Engineering, Qazvin branch, \\ Islamic Azad University, Qazvin, Iran \\ ${ }^{2}$ Islamic Azad University, South Tehran Branch, Department of Computer, Tehran, Iran
}

\begin{abstract}
Character recognition is a new research field in the domain of pattern recognition which deals with the style of writing. Some of the challengeable problems in character identification are changing in the style of writing, font and turns of words and etc. In this paper, the goal is Persian character identification using independent orthogonal moment as the feature extraction technique.The proposed feature extraction method is the combination of Pseudo-Zernike Moment and Fourier-Mellin Moment called Pseudo-ZernikeMellin Moment to extract feature vector from Persian characters. The proposed character identification system is evaluated on the HODA dataset and obtained $97.76 \%$ acceptance rate.
\end{abstract}

\section{KEYWORDS}

Character Recognition, Pseudo-Zernike Invariant Moments, Fourier-Mellin moments, Back Propagation (BP), K-Nearest Neighbor( $k$-NN),RadialNeuralNetwork (RBF).

\section{INTRODUCTION}

Automated recognition of text is one of best and attractive research field in the domain of pattern recognition[1]. In this domain, handwriting recognition is considered as the transformation of the text presented in the spatial from of graphical marks into its symbolic representation.This process is applicable in marking digital copies of handwriting documents and in many automated processing takes such as automated mail sorting.In automated mail sorting, letters are directed to the correct location usingidentification of the handwritten address. The handwriting recognition technique is divided into two different approaches, on-line and off-line[2].

In the on-line method, recognition of handwriting is done on the characters captured using an input device such as digitized pen [3] while in the off-line method, identification is performed on the image a character [4].

In this paper, the progress of character identification is as follows: at first, the input image of a character is captured and then the feature extraction step is performed on the input image in order to extract feature vector. After that the extracted feature vector is utilized in classification step to identify and classify the character contained in the input image.

This paper is organized as follow: Section 2 shows the feature extraction step of a character recognition system. In Section 3, the proposed method is described. Section 4 demonstrates experimental results.

DOI : 10.5121/ijcsity.2014.2201 
International Journal of Computational Science and Information Technology (IJCSITY) Vol.2, No.2, May 2014

\section{Feature EXTraction}

In the character recognition system three steps should be passed: pre-processing, feature extraction and classification. In the feature extraction step, a vector which includes impressive information is produced from input character image in order to make an efficient decision on the classification of input image. In the feature extraction phase, the main challenge is that the feature extraction method is not public and depends on the application. In the domain of feature extraction, methods are categorized into two groups:structural features and statistical features [56]. The first group is based on the local structure of image. In the other words, the structural features are performed on the local data.

In the second group, the feature vector is created according to the global data.The feature extraction techniques which are based on the statistical approach are Principle Component Analysis (PCA), Legender Moment (LM) [7] and Pseudo-Zernike Moments (PZM) [8] and Fourier-Mellin Moment (FMM) [9]. Legendre functions are Legendre differential equation. Some profits of Legendre moments are orthogonallity, independency of moments from each other and freedom of data redundancy. In the proposed method, the combination of PZM and FMM for the character recognition is considered.

\section{Proposed Method}

Previous feature extraction methods shows poor performance on the input image because of existence of noise in image [10], image rotation [11] and image resizing [10].

The goal of this paper is improving of two important steps, feature extraction and classification steps. For this purpose, at first, combination of Pseudo-Zernike and Fourier Mellin moments as the feature extraction method is proposed. The combined approach has overcome on the disadvantages of the previous methods: for the image rotation,Pseudo-Zernike extracts statistical features which are independent of the scaling and rotation. Also unlike the geometrical-based feature extraction methods, statistical methods areindependent of image resizing. For the last above problem, the proposed method overcomes to the noise of image.

To improve the classification step of character identification system, an improvement on $k$ Nearest Neighbor $(k-\mathrm{NN})$ is proposed that will be described as follow.

\subsection{Pseudo-Zernike Moment (PZM)}

PZM is geometric-based moment that is employed to extract features according to the global information [18]. The advantage of PZM is that its orthogonal momentsare shift, rotation, and scale invariants[4][12-14][17]. Pseudo-Zernike contains severalorthogonal sets of complexvalued polynomials defined as

$$
V_{n m}(x, y)=R_{n m}(x, y) \exp \left(j m \tan ^{-1}\left(\frac{y}{x}\right)\right)
$$

where $x^{2}+y^{2} \leq 1, n \geq 0,|m| \leq n$, and the radial polynomials $\left\{R_{n m}\right\}$ are defined as

$$
R_{n m}(x, y)=\sum_{s=0}^{(n-|m|)} D_{n,|m|, s}\left(x^{2}+y^{2}\right)^{\frac{n-s}{2}}
$$

where

$$
D_{n,|m|, s}=(-1)^{s} \frac{(2 n+1-s) !}{s !(n-|m|-s) !(n-|m|-s+1) !}
$$


International Journal of Computational Science and Information Technology (IJCSITY) Vol.2, No.2, May 2014 The PZM of order $n$ and repetition $m$ can be computed as

$$
P Z M_{n m}=\frac{n+1}{\pi} \sum_{x} \sum_{y} f(x, y) V_{n m}^{*}(x, y)
$$

It should be noted that the PZMis computed for positive $m$ because $V_{n m}(x, y)=V_{n m}^{*}(x, y)$.In the rotated image, if the absolute value or value of PZM is considered as the feature,the feature is independent of rotation [17].

\subsection{Fourier-Mellin Moments (FMMs)}

The orthogonal Fourier-Mellin Moments (FMMs) are proposed by Sheng and Sheng [9] which they are suitable for scale and rotation-invariant pattern recognition. The orthogonal FMMs are the generalization of Zernike moments and orthogonalized complex moments. The orthogonal FMMs are defined as:

$\phi_{\mathrm{nl}}=\frac{\mathrm{n}+1}{\pi} \int_{0}^{2 \pi} \int_{0}^{1} Q_{\mathrm{n}}(\mathrm{r}) \mathrm{e}^{-\mathrm{il} \varphi} \mathrm{f}(\mathrm{r}, \varphi) \mathrm{rdr} \mathrm{d} \varphi \mathrm{n}=0,1,2, \ldots, \mathrm{l}=0, \pm 1, \pm 2, \ldots(5)$

where $f(r, \varphi)$ is the image and the radial function $Q_{n}(r)$ is calculated as:

$$
Q_{\mathrm{n}}(\mathrm{r})=\sum_{\mathrm{s}=0}^{\mathrm{n}}(-1)^{\mathrm{n}+\mathrm{s}} \frac{(\mathrm{n}+\mathrm{s}+1) !}{(\mathrm{n}-\mathrm{s}) ! \mathrm{s} !(\mathrm{s}+1) !} \mathrm{r}^{\mathrm{s}}
$$

The first six polynomials of the moments are shown as follows:

$Q_{0}(r)=1$,

$Q_{1}(r)=-2+3 r$,

$Q_{2}(r)=3-12 r+10 r^{2}$,

$Q_{3}(r)=-4+30 r-60 r^{2}+35 r^{3}$,

$Q_{4}(r)=5-60 r+210 r^{2}-280 r^{3}+126 r^{4}$,

$Q_{5}(r)=-6+105 r-500 r^{2}+1260 r^{3}-1260 r^{4}+462 r^{5} \cdot(7)$

The main difference between orthogonal FMM and PZM is absence of restrictions on $n \geq l$ and also independence of the radial function $\mathcal{Q}_{n}(r)$ in the repetition of factor $l$.

\subsection{Creating feature vector}

In this step, the two main feature extractors, PZMand Fourier-Mellin moments, are employed separately to extract feature vector.The feature vector extracted by PZM are defined as

$F V_{-} P Z M_{j}=\left\{P Z M_{k m} \mid k=j, j+1, \ldots, N\right\}$

where $j$ is interval $[1, N-1]$ and so, $F V_{j}$ contains all the PZM from order $j$ to $N$. In Table 1 , samples of feature vector elements is shownfor $j=3,5$ and 9 , and $N=10$. According the table, increasing of $j$ decreases the number of elements in each feature vector $\left(F V_{j}\right)$. 


\begin{tabular}{|c|c|c|c|}
\hline \multirow{2}{*}{$j$ value } & \multicolumn{2}{|c|}{$\mathrm{FV}{ }_{\mathrm{j}}$ feature elements $\left(P Z M_{k m}\right)$} & \multirow{2}{*}{$\begin{array}{l}\text { Number of } \\
\text { feature element }\end{array}$} \\
\hline & $\mathbf{K}$ & $\mathbf{M}$ & \\
\hline \multirow{7}{*}{4} & 4 & $0,1,2,3,4$ & \multirow{7}{*}{56} \\
\hline & 5 & $0,1,2,3,4,5$ & \\
\hline & 6 & $0,1,2,3,4,5,6$ & \\
\hline & 7 & $0,1,2,3,4,5,6,7$ & \\
\hline & 8 & $0,1,2,3,4,5,6,7,8$ & \\
\hline & 9 & $0,1,2,3,4,5,6,7,8,9$ & \\
\hline & 10 & $0,1,2,3,4,5,6,7,8,9,10$ & \\
\hline \multirow{5}{*}{6} & 6 & $0,1,2,3,4,5,6$ & \multirow{5}{*}{45} \\
\hline & 7 & $0,1,2,3,4,5,6,7$ & \\
\hline & 8 & $0,1,2,3,4,5,6,7,8$ & \\
\hline & 9 & $0,1,2,3,4,5,6,7,8,9$ & \\
\hline & 10 & $0,1,2,3,4,5,6,7,8,9,10$ & \\
\hline \multirow{2}{*}{9} & 9 & $0,1,2,3,4,5,6,7,8,9$ & \multirow{2}{*}{21} \\
\hline & 10 & $0,1,2,3,4,5,6,7,8,9,10$ & \\
\hline
\end{tabular}

For FMM, the feature vector is obtained as:

$F V_{-} F M M=\left\{\phi_{\mathrm{nl}} \mid n=0,1,2, \ldots, l=0, \pm 1, \pm 2, \ldots\right\}$

The final feature vector is the combination of $F V_{-} P Z M_{j}$ and $F V_{-} F M M$ which contains all necessary information for detection of characters.

\subsection{Classification Using Improved $k$-Nearest Neighbor $(k$-NN)}

The final stage of handwriting system is the classification of character using the feature vector extracted from the previous step. In this step, the specific character shown in the input image will be detected. For this purpose, an improvement on the $k$-NN classifier which is called Improved $k-\mathrm{NN}$ is considered to enhance the accuracy of the classifier in character identification. In the simple $k$-NN, $k$ determines the number of samples as the neighbors of test sample and so for this variable, only one value is set. After that, the classification task is performed according to the Euclidean distance between the test sample and itsneighbors:

$$
\mathrm{d}=\sqrt{\sum_{\mathrm{i}=1}^{\mathrm{m}}\left(\mathrm{x}_{\mathrm{i}}-\mathrm{y}_{\mathrm{i}}\right)^{2}}
$$

After the calculation of distances, $k$-nearest neighbors of test sample are selected and then the test sample is classified to the class which covers the most of the neighbors.

The simple $k$-NN uses just one value for the variable $k$ which has bad effect on the performance of the classifier and so decreases its accuracy. Also, the simple $k-\mathrm{NN}$ is sensitive to the noisy data because of single value for the variable. It means that the simple $k$-NN makes randomly decision on the class of the test sampleso, the performance of $k$-NN classifier is decreased.

In the Improved $k$-NN, a set (interval) of integer values is considered for variable $k$ and so a class is assigned to the test sample per each value of the set. Finally, the class with the highest frequency of selection is chosen as the main (final) class of the test sample. For instance, suppose that four values $3,5,7$ and 9 is determined for the $k$. To classify test sample $A$, the distance 
International Journal of Computational Science and Information Technology (IJCSITY) Vol.2, No.2, May 2014

between $A$ and its neighbors is computed and for $k=3$ three nearest of its neighbors are selected in order to determine the class of $A$. After that this process is repeated for $k=5,7$ and 9 , and the class with the maximum frequency of selection is considered as the class of sample test $A$. This idea increases the accuracy of $k$-NN classifier. Unlike the simple $k$-NN, the results of Improved $k$-NN is produced without randomness and the most reliable results are obtained using Improved $k$-NN. Also, the Improved $k$-NN is robust to noisy data. The other advantage of Improved $k$-NN is that the runtime of character detection process is reduced in the proposed method and so the proposed method will converge faster to optimal solution.

\section{EXPERIMENTAL RESULTS}

In this section, the proposed character recognition system is evaluated. In this regard, the proposed hybrid Pseudo-Zernike-Mellin Moment is compared with both of the PZM and FMM on the detection rate, speed and the size of feature vector. Also, the Improved $k$-NN classifier is compared with two neural networks such as Radial Basis Function (RBF), Back Propagation (BP) on the accuracy and runtime.

In this paper, the testing phase is utilized on the HODA dataset [15]which contains the number styles " 0 " to " 9 ". The total number of used images of number is 100 in size of $13 \times 13$ with different forms of handwriting.

At the first step, the PZM, FMM and Pseudo-Zernike-Mellin Moments as the feature extractors are compared on the runtime which is demonstrated in Table 2. In this table, the effect of feature vectors with different size is mentioned. The runtime is increased when the size of feature vector is increased. The results show the success of hybrid Pseudo-Zernike-Mellin because of the parallel processing of PZM and FMM in feature extraction and so a combined feature vector is extracted in the minimum runtime.

In Table 3, identification speed of Improved $k$-NN is compared with the BP neural network. According to the table, the classifiers are compared in different size of feature vector and the achieved results proves that the Improved $k$-NN takes best scores because of feature extracted from the hybrid Pseudo-Zernike-Mellin Moment. The graphical results of Table 3 are shown in Figure 1. It should be noted that in this test the results of BP and RBF neural network are very similar to each other and theresults of BP is just presented.

Next, the impact of each individual moment, PZM, FMM and hybrid Zernike-Mellin, on the classification step is examined. In Table 4, the results prove the high impact of feature vector extracted by the PZM to the Improved $k-\mathrm{NN}$ algorithm because of usage of multi-level neighborhood rather than a value.In the second place, the best results are obtained using BP network, and RBF neural network is located on the third level.

In Table 5, the previous testing phase is done using the feature vector elements extracted from FMM. In this table, the best results are produced by proposed classifier. It should be noted that the results using PZM is higher than the FMM ones that shows the superiority of PZM to FMM in creation of informative feature vector.

In Table 6, the effect of feature vector extracted using hybrid Pseudo-Zernike-Mellin Moment on the classification step.According to the table, the best results are achieved using Improved $k$-NNin comparison the other method. As a result on Tables 4, 5 and 6, the Improved $k$-NN using hybrid Pseudo-Zernike-Mellin Moment shows the best performancein comparison to the other classifiers and also with PZM and FMM feature extractors. The reason of success of the proposed scheme refers to the most informative feature vector extracted by the hybrid Pseudo-ZernikeMellin Moment, and the classification of Improved $k$-NN with high confidence (without randomness). 
International Journal of Computational Science and Information Technology (IJCSITY) Vol.2, No.2, May 2014

Table 2. Comparison of PZM, FMM and Pseudo-Zernike-Mellin Moment on the runtime. Suppose that $N=17$. The bold values show the best obtained results. FE means Feature Element.

\begin{tabular}{|c|c|c|c|}
\hline No. of FE & PZM & FMM & $\begin{array}{c}\text { Pseudo-Zernike- } \\
\text { Mellin Moment }\end{array}$ \\
\hline 3 & 0.003 & 0.003 & $\mathbf{0 . 0 0 2}$ \\
\hline 4 & 0.005 & 0.006 & $\mathbf{0 . 0 0 4}$ \\
\hline 5 & 0.007 & 0.006 & $\mathbf{0 . 0 0 5}$ \\
\hline 6 & 0.01 & 0.011 & $\mathbf{0 . 0 0 6}$ \\
\hline 7 & 0.024 & 0.013 & $\mathbf{0 . 0 1 2}$ \\
\hline 10 & 0.04 & 0.014 & $\mathbf{0 . 0 1 7}$ \\
\hline 13 & 0.07 & 0.05 & $\mathbf{0 . 0 2 4}$ \\
\hline 15 & 0.11 & 0.08 & $\mathbf{0 . 0 3 2}$ \\
\hline 17 & 0.13 & 0.08 & $\mathbf{0 . 0 4 1}$ \\
\hline 25 & 0.19 & 0.17 & $\mathbf{0 . 1 4}$ \\
\hline
\end{tabular}

The last phase of testing is evaluation of the proposed method against the "Salt \& Pepper" and "Gaussian" noise distributions on the input image of characters. The results of previous classifiers for the detection of characters on the noisy images with "Salt \& Pepper" and "Gaussian" distributions are reported in Tables 7 and 8, respectively. Note that for theboth of the noise distributions, the utilized feature extraction method is hybrid Pseudo-Zernike-Mellin Moment. As can be seen in Tables 7 and 8, the Improvedk-NN shows the best results. These tables demonstrate that the proposed classifier has significant improvement on the behaviour of simple $k$-NN because the simple $k$-NN is extremely sensitive to the noisy data. In Figure 2, the graphical results of classifiers on detection of character in noisy imagewith Gaussian distribution are demonstrated. In the last phase, the results of Improved $k$-NN on the both of the "Salt \& Pepper" and "Gaussian" noise distributions are presented in Figure 3. According to this figure, the "Salt \& Pepper" noise has more impact on decreasing of the detection rate of Improved $k$-NN.

Table 3. Comparison of BP and Improved $k$-NN classifiers on the detection of characters on the runtime. Suppose $N=17$. The bold values show the best results. FE means Feature Element.

\begin{tabular}{|c|c|c|}
\hline No. of FE & BP & Improved $k$-NN \\
\hline 2 & 0.399 & $\mathbf{0 . 3 7 8}$ \\
\hline 3 & 0.339 & $\mathbf{0 / 3 8 0}$ \\
\hline 4 & 0.402 & $\mathbf{0 . 3 7 4}$ \\
\hline 5 & 0.429 & $\mathbf{0 . 3 7 8}$ \\
\hline 6 & 0.450 & $\mathbf{0 . 3 9 9}$ \\
\hline 8 & 0.557 & $\mathbf{0 . 4 0 7}$ \\
\hline 10 & 0.664 & $\mathbf{0 . 4 2 8}$ \\
\hline 13 & 0.811 & $\mathbf{0 . 4 5 2}$ \\
\hline 15 & 1.03 & $\mathbf{0 . 4 5 9}$ \\
\hline 17 & 1.311 & $\mathbf{0 . 5 2 3}$ \\
\hline 23 & 1.978 & $\mathbf{0 . 7 8 2}$ \\
\hline 25 & 2.20 & $\mathbf{0 . 9 1 6}$ \\
\hline
\end{tabular}


International Journal of Computational Science and Information Technology (IJCSITY) Vol.2, No.2, May 2014

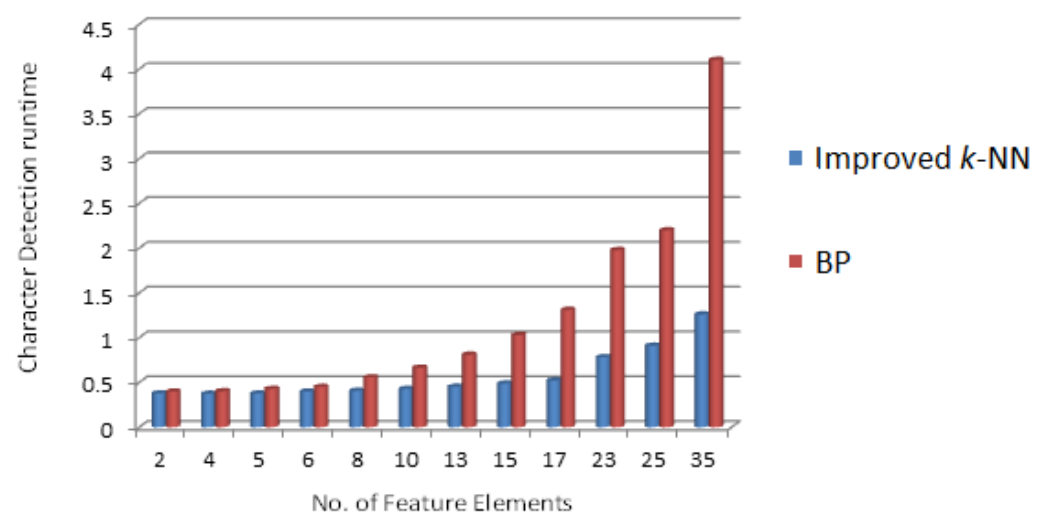

Figure 1. Comparison of BP and Improved $k$-NN classifiers on Character detection runtime

Table 4.Comparison of BP, RBF and Improved $k$-NN classifiers on the accuracy based on the feature vector extracted by PZM. Suppose $N=17$. The bold values show the best results. FE means Feature Element.

\begin{tabular}{|c|c|c|c|}
\hline No. of FE & BP & RBF & Improved k-NN \\
\hline 3 & 87.1 & 65.2 & $\mathbf{9 0 . 2}$ \\
\hline 4 & 87.4 & 65.9 & $\mathbf{9 0 . 5}$ \\
\hline 5 & 87.9 & 66.2 & $\mathbf{9 0 . 9}$ \\
\hline 6 & 88.1 & 68.3 & $\mathbf{9 0 . 9}$ \\
\hline 8 & 88.2 & 69.8 & $\mathbf{9 2 . 3}$ \\
\hline 10 & 88.2 & 74.3 & $\mathbf{9 3 . 7}$ \\
\hline 13 & 88.5 & 81.8 & $\mathbf{9 4 . 3}$ \\
\hline 15 & 88.7 & 86.2 & $\mathbf{9 4 . 5}$ \\
\hline 17 & 88.9 & 87.7 & $\mathbf{9 5 . 6}$ \\
\hline 23 & 90.1 & 88.3 & $\mathbf{9 6 . 2 5}$ \\
\hline
\end{tabular}

Table 5.Comparison of BP, RBF and Improved $k$-NN classifiers on the accuracy based feature extracted by FMM. Suppose that $N=17$. The bold values show the best obtained results. FE means Feature Element.

\begin{tabular}{|c|c|c|c|}
\hline No. of FE & BP & RBF & Improved $k$-NN \\
\hline 3 & 87.4 & 80.1 & $\mathbf{8 9 . 1}$ \\
\hline 4 & 87.3 & 80.2 & $\mathbf{8 9 . 5}$ \\
\hline 5 & 88.0 & 81.9 & $\mathbf{8 9 . 9}$ \\
\hline 6 & 88.1 & 83.4 & $\mathbf{9 0 . 5}$ \\
\hline 8 & 88.5 & 85.4 & $\mathbf{9 1 . 3}$ \\
\hline 10 & 88.8 & 86.6 & $\mathbf{9 1 . 5}$ \\
\hline 13 & 89.6 & 87.2 & $\mathbf{9 2 . 7}$ \\
\hline 15 & 89.7 & 87.9 & $\mathbf{9 3 . 2}$ \\
\hline 17 & 89.9 & 88.1 & $\mathbf{9 3 . 8}$ \\
\hline 23 & 90.4 & 89.1 & $\mathbf{9 6 . 2}$ \\
\hline 25 & 91.5 & 90.4 & $\mathbf{9 6 . 8}$ \\
\hline
\end{tabular}


International Journal of Computational Science and Information Technology (IJCSITY) Vol.2, No.2, May 2014

Table 6. Comparison of BP, RBF and Improved $k$-NN classifiers on the accuracy based feature extracted by hybrid Pseudo-Zenike-Mellin Moment. Suppose that $N=17$. The bold values show the best obtained results.

FE means Feature Element.

\begin{tabular}{|c|c|c|c|}
\hline No. of FE & BP & RBF & Improved k-NN \\
\hline 3 & 88.6 & 85.6 & $\mathbf{9 0 . 1}$ \\
\hline 4 & 88.7 & 86.3 & $\mathbf{9 0 . 5}$ \\
\hline 5 & 89.1 & 86.9 & $\mathbf{9 1 . 3}$ \\
\hline 6 & 89.5 & 87.2 & $\mathbf{9 1 . 5}$ \\
\hline 8 & 89.9 & 87.5 & $\mathbf{9 1 . 9}$ \\
\hline 10 & 90.2 & 87.9 & $\mathbf{9 3 . 5}$ \\
\hline 13 & 90.5 & 88.1 & $\mathbf{9 4 . 8}$ \\
\hline 15 & 90.9 & 88.6 & $\mathbf{9 5 . 9}$ \\
\hline 17 & 91.5 & 88.9 & $\mathbf{9 6 . 2}$ \\
\hline 23 & 91.9 & 90.1 & $\mathbf{9 7 . 9}$ \\
\hline 25 & 92.3 & 90.2 & $\mathbf{9 8 . 1}$ \\
\hline
\end{tabular}

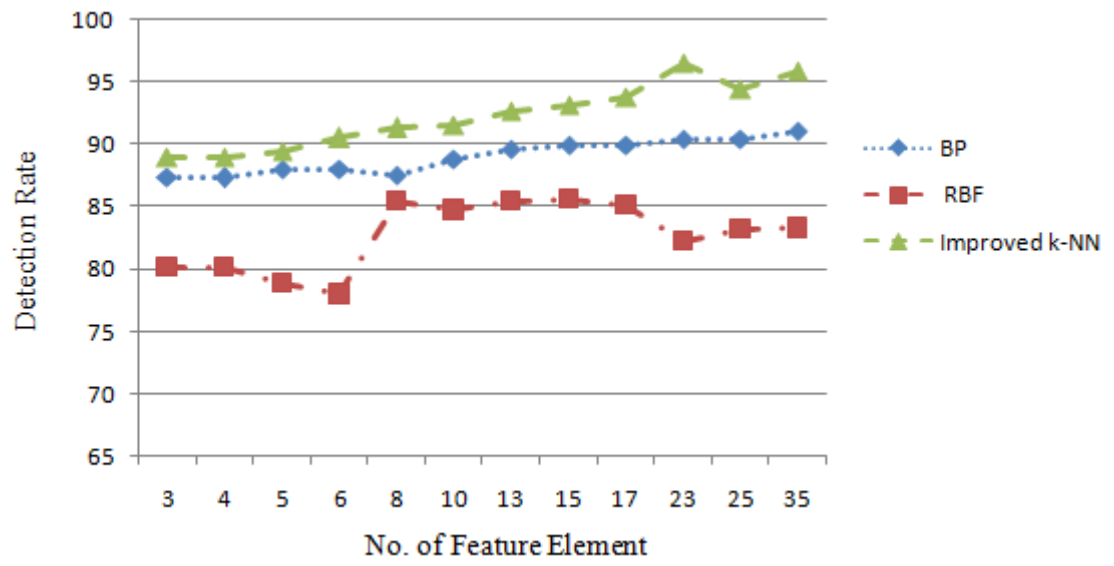

Figure 2. Comparison of BP, RBF and Improved $k$-NN classifiers on Character detection runtime

Table 7. Comparison on the detection rate of BP, RBF and Improved $k$-NN classifiers on the "Salt \& Pepper" noisy images. Suppose $N=17$ and bold values show the best results.

\begin{tabular}{|c|c|c|c|}
\hline Noise frequency $(\%)$ & BP & RBF & Improved $k$-NN \\
\hline 0.01 & $\mathbf{8 4 . 3}$ & 85.7 & $\mathbf{8 7 . 2}$ \\
\hline 0.02 & $\mathbf{8 3 . 3}$ & 84.9 & $\mathbf{8 7 . 1}$ \\
\hline 0.03 & $\mathbf{8 2 . 4}$ & 83.4 & $\mathbf{8 7 . 1}$ \\
\hline 0.04 & $\mathbf{8 2 . 2}$ & 82.8 & $\mathbf{8 6 . 5}$ \\
\hline 0.05 & $\mathbf{8 1 . 9}$ & 82.6 & $\mathbf{8 5 . 4}$ \\
\hline 0.06 & $\mathbf{8 0 . 3}$ & 81.4 & $\mathbf{8 4 . 3}$ \\
\hline
\end{tabular}


International Journal of Computational Science and Information Technology (IJCSITY) Vol.2, No.2, May 2014

Table 8. Comparison on the detection rate of BP, RBF and Improved k-NN classifiers on the "Gaussian" noisy images. Suppose $N=17$ and bold values show the best results.

\begin{tabular}{|c|c|c|c|}
\hline Noise frequency $(\%)$ & BP & RBF & Improved k-NN \\
\hline 0.01 & 81.1 & 82.3 & $\mathbf{8 5 . 6}$ \\
\hline 0.02 & 80 & 81.8 & $\mathbf{8 5 . 2}$ \\
\hline 0.03 & 80.8 & 81.5 & $\mathbf{8 4 . 9}$ \\
\hline 0.04 & 78.3 & 81.7 & $\mathbf{8 3 . 6}$ \\
\hline 0.05 & 80.8 & 81.3 & $\mathbf{8 3 . 4}$ \\
\hline 0.06 & 79.7 & 80.7 & $\mathbf{8 3 . 1}$ \\
\hline
\end{tabular}

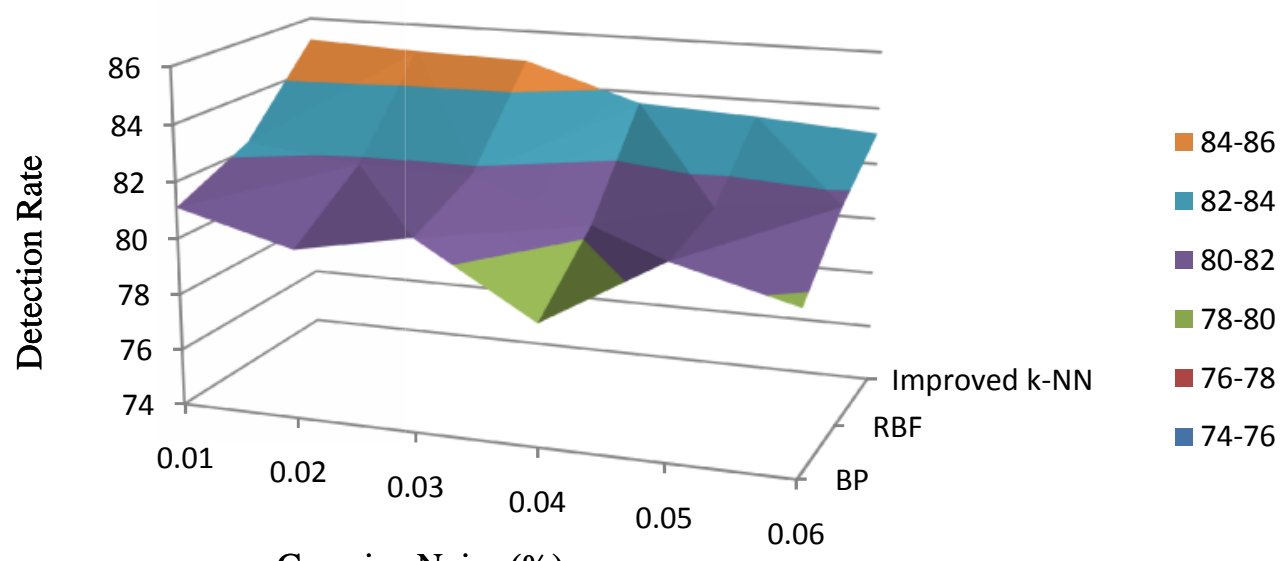

Figure 3. Comparison of $\mathrm{BP}, \mathrm{RBF}$ and Improved $k$-NN classifiers on Character detection runtime

\section{CONCLUSION}

In this paper, a new approach is proposed for handwriting recognition. The proposed method has two innovations. The first innovation is on the feature extraction step where the hybridization of PZD and FMM which is called hybrid pseudo Zernike-mellin moment. In the second innovation, an improvement on the simple K-NN is proposed where for the variable $\mathrm{K}$, a multi-value neighborhood rather than a value is set.The proposed system is evaluated on HODA dataset which contains non-noisy and noisy images. The results show that the proposed method is able to detect the characters with $97.76 \%$ detection rate in the reduced time.

\section{REFERENCES}

[1] Khosavi, H., Kabir, E., (2007) "Introducing a very large dataset of handwritten Farsi digits and a study on their varieties", Pattern Recognition Letters, Vol. 28, No. 10, pp. 1133-1141.

[2] de Campos, T., Babu, B. R., \&Varma, M. (2009) "Character recognition in natural images".

[3] Harmon, L. D. (1972) "Automatic recognition of print and script", Proceedings of the IEEE, Vol. 60, No.10, pp. 1165-1176. 
International Journal of Computational Science and Information Technology (IJCSITY) Vol.2, No.2, May 2014

[4] Broumandnia, A., Shanbehzadeh, J. (2007) "Fast Zernike wavelet moments for Farsi character recognition”, Image Vision Computing, Vol. 25, No. 5, pp. 717-726.

[5] Broumandnia, A., Shanbehzadeh, J., Nourani, M. (2007) "Handwritten Farsi/Arabic Word Recognition", AICCSA 2007, pp. 767-771.

[6] Broumandnia, A., Shanbehzadeh, J., RezakhahVarnoosfaderani, M. (2008) "Persian/arabic handwritten word recognition using M-band packet wavelet transform", Image Vision Computing, Vol. 26, No. 6, pp. 829-842.

[7] Liao, S. \&Pawlak, M. (1996) "On image analysis by moments", IEEE Transactions on Pattern Analysis and Machine Intelligence, Vol. 18, No. 3, pp. 254-266.

[8] Zernike, v.F. (1934), "Beugungstheorie des schneidenver-fahrens und seiner verbesserten form, der phasenkontrastmethode”, Physica, Vol. 1, No. 7, pp. 689-704.

[9] Sheng, Y., \&Shen, L. (1994) "Orthogonal Fourier-Mellin moments for invariant pattern recognition", JOSA A, Vol. 11, No. 6, pp. 1748-1757.

[10] Altman, N. S. (1992) "An introduction to kernel and nearest-neighbor nonparametric regression", The American Statistician, Vol. 46, No. 3, pp. 175-185.

[11] Abdi. H., \& Williams, L.J. (2010) "Principal component analysis", Wiley Interdisciplinary Reviews: Computational Statistics, Vol. 2, pp. 433-459.

[12] Belhumeur, P., Hespanha, J. \&Kriegman, D. (1997) "Eigenfaces vs. fisherfaces recognition using class specific linear projection", IEEE Transactions on Pattern Analysis and Machine Intelligence, Vol. 19, No. 7, pp. 711-720.

[13] Belkasim, S., Shridhar, M. \&Ahmadi, M. (1991) "Pattern recognition with moment invariants: A comparative study and new results", Pattern Recognition, Vol. 24, No.12, pp. 1117-1138, http://www.sciencedirect.com/science/article/pii/003132039190140Z.

[14] Teh, C.H. \& Chin, R. (1988) “On image analysis by the methods of moments". IEEE Transactions on Pattern Analysis and Machine Intelligence, Vol. 10, No. 4, pp. 496-513.

[15] Free and Commercial Resources for Farsi OCR, http://www.farsiocr.ir/

[16] Bailey, R.R. (1993) "Automatic recognition of handwritten numerals via orthogonal moments using statistical and neural network classifiers", Ph.D. thesis, Dallas, TX, USA (1993), aAI9331109.

[17] Haddadnia, J., Ahmadi, M. \&Faez, K. (2002) "An efficient method for recognition of human faces using higher orders pseudo Zernike moment invariant”, In: Proceedings. Fifth IEEE International Conference on Automatic Face and Gesture Recognition 2002, pp. 330-335.

[18] Haddadnia, J., Faez, K. \&Moallem, P. (2001) "Neural network based face recognition with moment invariants", In Proceedings of Int. Conference on Image Processing 2001, Vol. 1, pp. 1018-1021. 